\title{
Procédés textiles : la place de la rhéologie
}

\author{
Dominique Dupuis ${ }^{\mathrm{a}}$
}

Laboratoire de Physique et Mécanique Textiles UMR 7189 CNRS-UHA, École Nationale Supérieure d'Ingénieurs Sud Alsace, Université de Haute Alsace, 11 rue Alfred Werner, 68093 Mulhouse Cedex, France

Reçu le 28 août 2008, accepté le 9 avril 2009

Résumé - Les différentes étapes de fabrication et de transformation du matériau textile mettent en jeu des fluides non newtoniens dont les propriétés rhéologiques doivent être maîtrisées. Cet article traite principalement de deux exemples relevant du domaine de l'ennoblissement : l'enduction et l'impression de tissus. Les résultats présentés apportent des éléments de compréhension du fonctionnement de procédés faisant intervenir des fluides de formulation très complexe.

Mots clés : Rhéologie / solutions de polymères / ennoblissement / enduction / impression / épaississants

Abstract - Textile processes: the importance of rheology. The various stages of transformation of the textile material involve non Newtonian fluids. The knowledge of their rheological properties is of great importance to control the efficiency of the process. Two examples in the field of finishing are considered in this paper: the blade coating and the printing of fabrics. The results contribute to a better understanding of process where complex formulated fluids are used.

Key words: Rheology / polymer solutions / finishing / blade coating / textile printing / thickeners

\section{Nomenclature}

\begin{tabular}{|ll|}
\hline$h:$ & hauteur de racle \\
$G^{\prime}:$ & module de conservation \\
$G^{\prime \prime}:$ & module de perte \\
$N_{1}:$ & première différence des contraintes normales \\
$P:$ & coefficient de pénétration \\
$T:$ & épaisseur de fluide entraînée (adimensionnelle) \\
$U:$ & vitesse relative racle-support \\
$W e:$ & nombre de Weissenberg \\
$\delta:$ & angle de perte \\
$\eta:$ & viscosité de cisaillement \\
$\theta:$ & angle d'inclinaison de la racle \\
$\sigma:$ & contrainte de cisaillement \\
$\omega:$ & pulsation \\
\hline
\end{tabular}

\section{Introduction}

De la fibre au produit fini tel qu'on le rencontre dans la vie quotidienne, le matériau textile subit plusieurs procédés de transformation où interviennent des écoulements de matière aux propriétés rhéologiques plus ou moins complexes. Les exemples qui seront considérés ici ne constituent pas à l'évidence une liste exhaustive.

\footnotetext{
a Auteur pour correspondance : dominique.dupuis@uha.fr
}

Le filage de polymère est à la base de la fabrication des fibres synthétiques. Il met en œuvre, généralement, des polymères à l'état fondu dont le comportement est non newtonien. Les écoulements mis en jeu sont principalement des écoulements élongationnels. À une autre échelle, un grand nombre de travaux concernent actuellement le filage électrostatique qui permet l'obtention de nano-filaments mais aussi de nano-composites. Le polymère est alors en solution dans un solvant et la solution est éventuellement chargée en particules [1].

Cependant, les différentes étapes d'élaboration du matériau textile supposent également l'utilisation de fluides auxiliaires. Citons par exemple l'encollage, opération préalable au tissage et qui consiste à imprégner la nappe de fils de chaîne d'une colle afin d'améliorer la résistance de ces fils aux sollicitations mécaniques imposées lors du tissage. Parmi les nombreux procédés d'ennoblissement, nous avons choisi de parler de l'enduction et de l'impression. Dans le premier cas, le tissu est enduit à l'aide d'une substance destinée à lui conférer des propriétés particulières, comme par exemple le rendre occultant à la lumière. La pâte d'enduction est alors généralement une solution de polymère ou encore une mousse éventuellement chargée en particules. Le tissu peut aussi être imprimé. Il existe différentes familles de procédés d'impression : au cadre, au rouleau, en jet d'encre... La formulation de la pâte ou de l'encre 


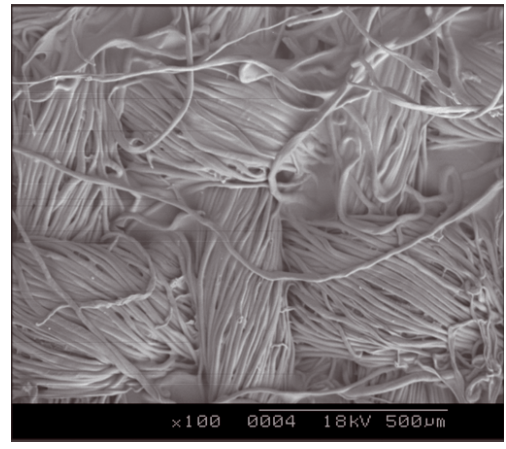

Fig. 1. Tissu enduit.

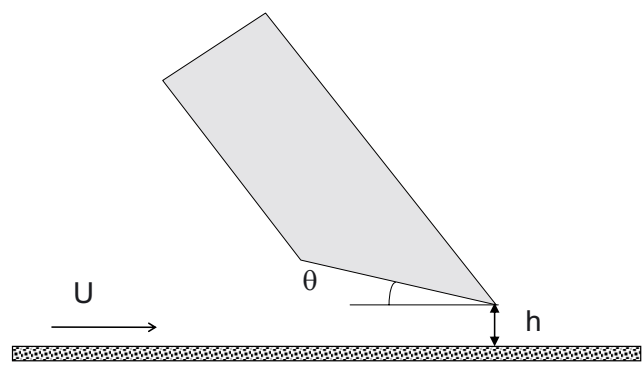

Fig. 2. Schéma du dispositif d'enduction.

d'impression fait intervenir plusieurs éléments ayant chacun une fonction bien spécifique pour l'efficacité du procédé.

Nous présentons ici quelques résultats d'études effectuées au sein de l'équipe physico-chimie des matériaux fibreux du LPMT dans les domaines de l'enduction et de l'impression.

\section{Enduction : influence de l'élasticité du fluide}

De manière générale, l'enduit doit recouvrir le tissu et bien adhérer (Fig. 1). Pour cela, il doit pénétrer dans les pores mais ne pas traverser l'étoffe pour ne pas être visible sur l'envers. Quelle est l'influence de la viscosité et de l'élasticité du fluide sur l'épaisseur déposée d'une part et sur la pénétration dans le tissu d'autre part?

Les expériences ont été réalisées à l'aide d'un dispositif d'enduction à racle schématisé sur la figure 2. Le support à enduire est animé d'une vitesse $U$ par rapport à la racle dont on peut faire varier la hauteur $h$ et l'angle d'inclinaison $\theta$. Les fluides utilisés sont différentes solutions de polymères modèles dont on peut contrôler les propriétés rhéologiques. Il s'agit d'une part de solutions aqueuses de carboxymethylcellulose (CMC) et de solutions aqueuses de polyacrylamide (PAA) à différentes concentrations. Ces solutions sont rhéofluidifiantes comme l'indiquent les courbes viscosité-gradient de vitesse de la figure 3. Par ailleurs, l'ajout d'une très faible quantité de PAA de masse molaire élevée à une solution de CMC ne modifie pratiquement pas sa viscosité de cisaillement, mais augmente considérablement son élasticité. Une solution de

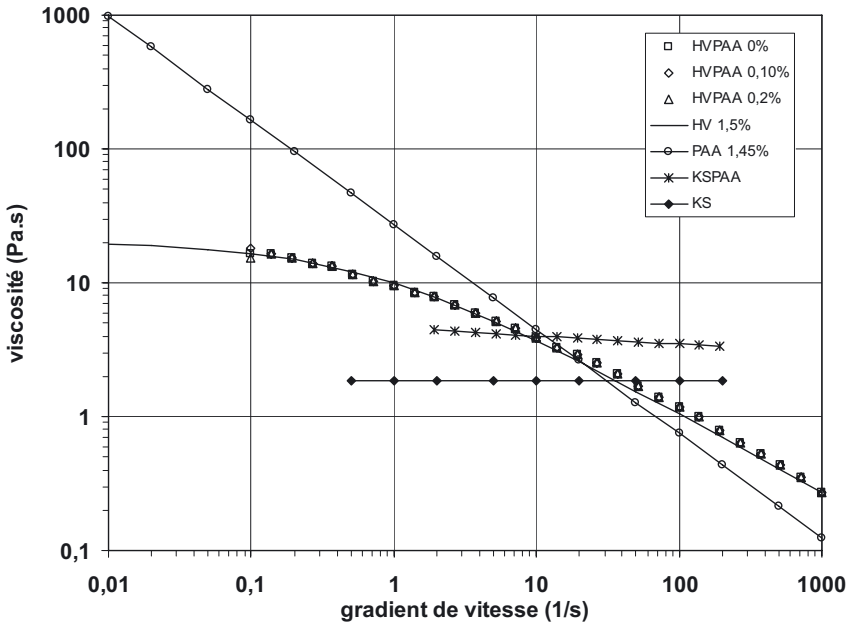

Fig. 3. Viscosité des différentes solutions de polymères.

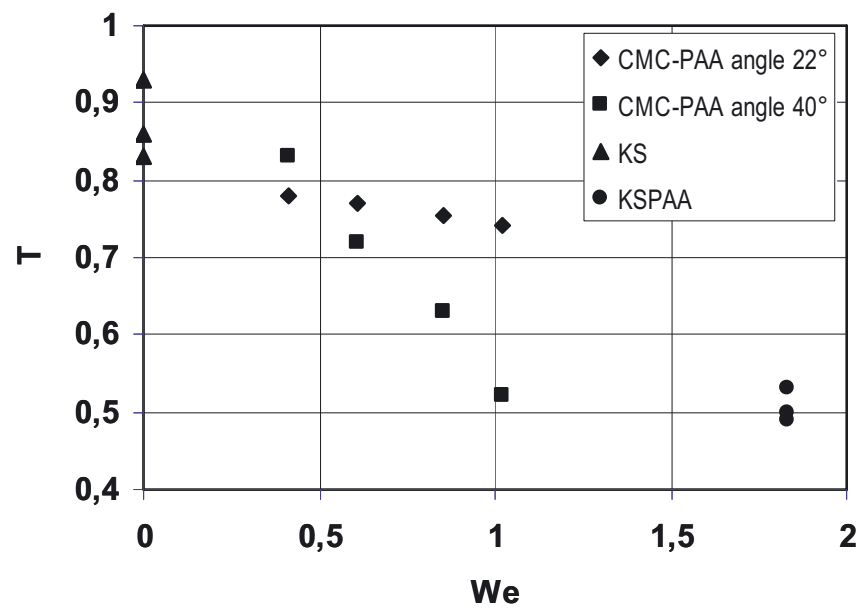

Fig. 4. Épaisseur de la couche de fluide en fonction du nombre de Weissenberg.

PAA (KSPAA) dans du sirop de maïs (KS) a également été utilisée. Elle a la particularité de présenter une viscosité pratiquement constante (sur la gamme de vitesses de cisaillement explorée) tout en étant très élastique. Le caractère élastique du fluide est mesuré par le nombre de Weissenberg $W e$ défini par $N_{1} / 2 \sigma$ où $N_{1}$ est la première différence des contraintes normales et $\sigma$ est la contrainte de cisaillement, à un gradient de vitesse donné. Les enductions sont réalisées sur deux types de supports : un film d'acétate de cellulose et une toile de coton. Dans le premier cas, il est possible de mesurer l'épaisseur de fluide déposé; dans le second, on s'intéresse au passage du fluide à travers le tissu. Le fluide est alors coloré et un coefficient de pénétration $P$ est défini à partir de mesures de réflectance à l'endroit et à l'envers du tissu.

La figure 4 représente l'épaisseur de la couche déposée $T$ (rapportée à la hauteur de lame $h$ ) en fonction du nombre de Weissenberg pour différents fluides et différents angles $\theta$. L'élasticité du fluide conduit à une diminution de l'épaisseur entraînée, diminution d'autant plus marquée que l'angle $\theta$ est plus important. Pour 

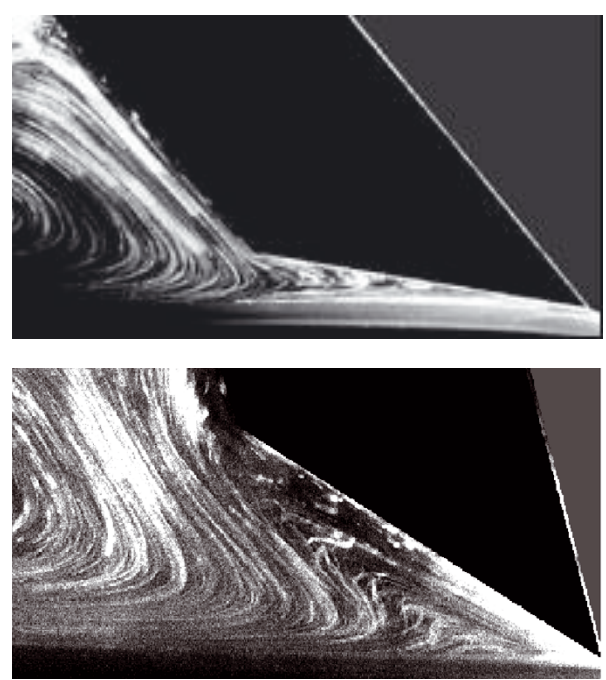

Fig. 5. En haut : KS, $\theta=8^{\circ}$; en bas : KS/PAA, $\theta=32^{\circ}[5]$.

l'échantillon KSPAA, les valeurs de $T$ sont faibles alors que pour le sirop de maïs (KS), elles sont en accord avec ce que prévoit la théorie sous l'hypothèse de l'approximation de la lubrification (dans la mesure où elle s'applique c'est-à-dire pour des angles pas trop grands).

Le comportement particulier du fluide KSPAA avait déjà été souligné dans la littérature [2] et différentes hypothèses avaient été formulées quant à l'écoulement sous la lame $[3,4]$. Des expériences de visualisation d'écoulement ont permis de mettre en évidence les lignes de courant lors du passage du fluide sous la racle (figure 5) [5]. Pour le fluide newtonien, avec un angle de $8^{\circ}$, on note l'existence d'un point d'arrêt sous la lame. Pour le fluide KSPAA, avec un angle de $32^{\circ}$, il apparaît d'importantes instabilités liées au développement des contraintes normales et conduisant à une faible épaisseur de fluide entraînée. La forme des lignes de courant est à rapprocher de celle qui avait été observée par Doremus et Piau [6] avec une autre géométrie et d'autres polymères et confirment les hypothèses avancées dans la littérature.

La figure 6 montre le coefficient de pénétration $P$ en fonction du nombre de Weissenberg $W e$ pour les solutions de PAA et pour les solutions CMC-PAA. Pour une famille de fluide donnée, $P$ diminue quand l'élasticité augmente. Dans le cas des solutions de PAA, une augmentation de $W e$ est liée à une augmentation de la concentration en polymère et donc à une augmentation de la viscosité. Les deux effets se combinent donc et la diminution de $P$ est importante. Les solutions CMC-PAA sont caractérisées par la même viscosité de cisaillement; c'est donc bien uniquement l'effet de l'élasticité qui est observé et qui conduit à une diminution de la pénétration.

\section{Impression : rhéologie des épaississants}

Le procédé d'impression étudié consiste en une succession de plusieurs étapes destinées à produire un dessin net, sans bavures et solide au lavage c'est-à-dire que

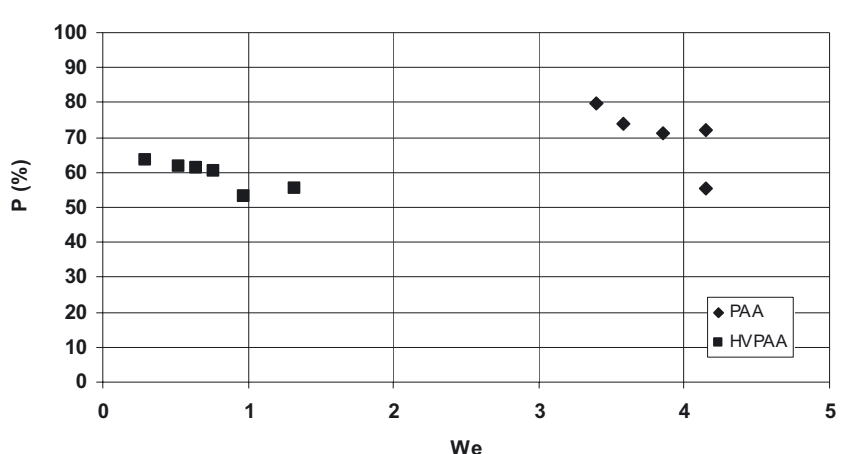

Fig. 6. Coefficient de pénétration en fonction du nombre de Weissenberg [7].

le colorant doit être fixé dans les fibres [8]. Le tissu est tout d'abord imprimé par l'intermédiaire d'un cadre (plat ou rotatif) puis, il est séché et stocké. La pâte d'impression contient, outre le colorant, des épaississants qui sont des mélanges de polysaccharides $[9,10]$. Le tissu passe ensuite dans un bain de foulardage qui contient un agent coagulant ayant pour fonction de «bloquer » l'impression avant les étapes ultérieures que sont le vaporisage et le finissage. Durant le vaporisage, l'épaississant et les fibres sont gonflés; il y a diffusion du colorant vers les fibres. Le finissage consiste en plusieurs opérations dont la fixation du colorant dans les fibres (oxydation et précipitation sous forme insoluble dans les fibres) et un savonnage pour éliminer le colorant non fixé et l'épaississant.

Lors de l'impression, la pâte est soumise à des contraintes pour pouvoir passer à travers les orifices du cadre. Lors du séchage, l'épaississant doit former un film assez souple pour ne pas casser lors des manipulations ultérieures du tissu. Enfin, la coagulation de l'épaississant doit conduire à la formation d'un gel « faible » susceptible de gonfler facilement. L'épaississant doit donc répondre à des contraintes multiples et sa formulation fait grandement appel au savoir-faire plus ou moins empirique de l'imprimeur.

La figure 7a montre la viscosité de cisaillement pour une dispersion aqueuse d'amidon de maïs éthérifié et pour une dispersion d'un mélange ternaire $(1,25 \%$ guaranate, $1,25 \%$ alginate, $2,5 \%$ amidon) à des concentrations de l'ordre de celles préconisées par le fabricant. À contrainte élevée, la viscosité est pratiquement la même tandis qu'à faible contrainte, l'utilisation d'un système ternaire supprime l'effet de seuil de contrainte observé avec l'amidon et réduit la thixotropie. Les mesures en oscillation (Fig. 7b) mettent en évidence le caractère nettement élastique de la dispersion d'amidon par rapport au système ternaire [11].

La coagulation qui intervient lors du foulardage résulte de la complexation des chaînes de guar contenues dans l'épaississant par des ions borates contenus dans le bain de foulardage [12]. Les associations interchaînes ainsi formées conduisent à une structure de réseau élastique aux propriétés de gel « faible » dépendant fortement du pH [13]. Ces types de gels ont des propriétés très particulières dans la mesure où les liaisons sont très 


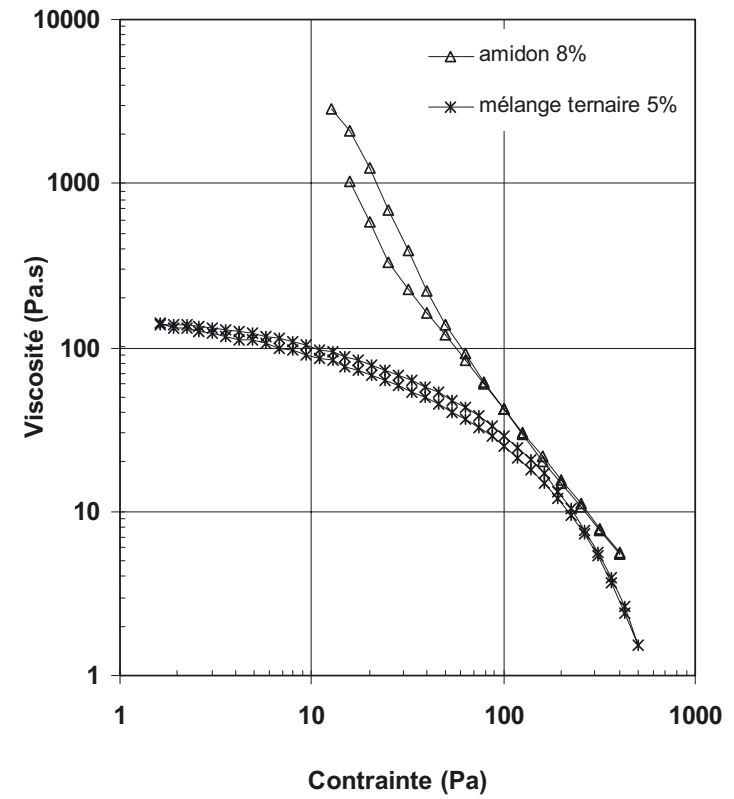

(a)

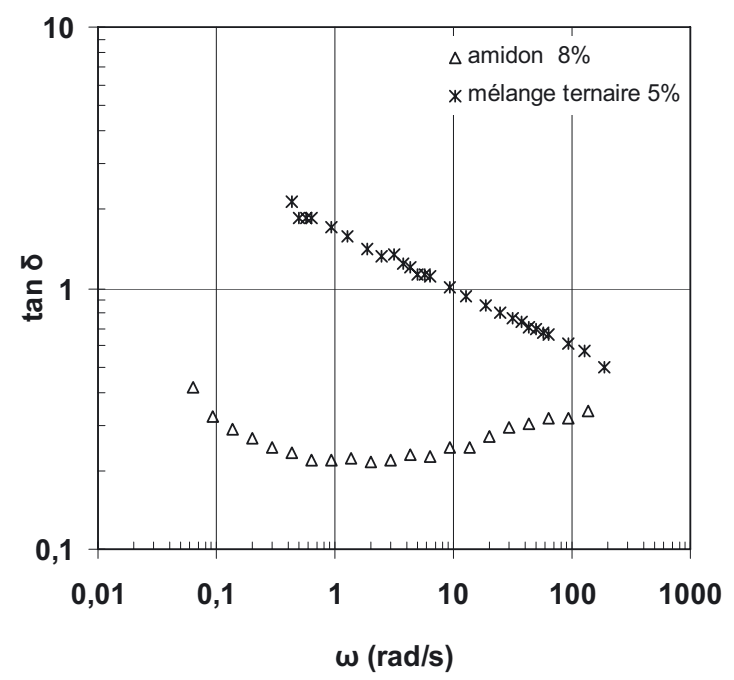

(b)

Fig. 7. (a) Viscosité de cisaillement, (b) $\tan \delta$ pour deux épaississants différents.

labiles [14]. La figure 8 montre les modules $G^{\prime}$ et $G^{\prime \prime}$ en fonction de la pulsation pour une dispersion de guar à différents $\mathrm{pH}$. À $\mathrm{pH} 5,5$ on obtient un comportement typique de celui d'une solution de polymère avec une zone terminale. Pour un $\mathrm{pH}$ supérieur à $8, G^{\prime}$ présente un plateau accompagné d'un minimum de $G^{\prime \prime}$. Là aussi, l'utilisation d'un mélange de polymères permet d'ajuster la force du gel [15].

\section{Conclusion}

Dans les procédés décrits ci-dessus, les fluides nonnewtoniens mis en œuvre sont soumis à des contraintes,

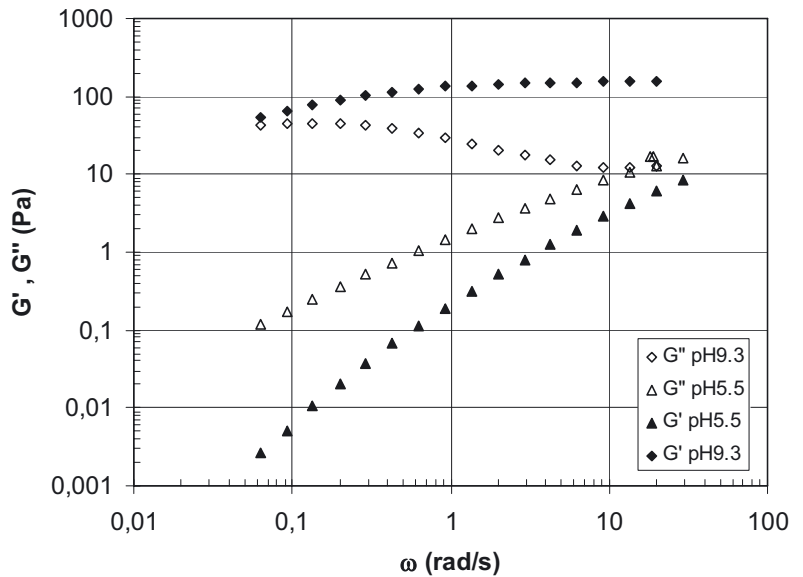

Fig. 8. $G^{\prime}$ et $G^{\prime \prime}$ pour une solution aqueuse de guaranate en présence d'acide borique.

ce qui modifie leurs propriétés d'écoulement. Il est cependant clair qu'en passant de l'échelle macroscopique à celle de la fibre, les propriétés aux interfaces vont jouer un rôle important en termes de mouillage et d'adhésion. L'exemple de l'encollage avec prémouillage est à ce titre riche d'enseignements. Le prémouillage d'un fil avec de l'eau augmente son énergie de surface et donc, favorise l'étalement d'une goutte de colle. De plus, la viscosité de cette colle diminue sous l'effet de contraintes mécaniques (solution de polymère rhéofluidifiante). Son étalement sur un film d'eau contribue par ailleurs à augmenter localement la dilution et donc à diminuer la viscosité. Tous ces effets vont dans le sens d'un meilleur étalement de la colle et donc d'une meilleure efficacité du procédé et d'une amélioration des propriétés mécaniques des fils [16]. Il ressort également de tout ceci que la compréhension des phénomènes mis en jeu nécessite une approche multiéchelle, allant de l'étoffe à la macromolécule, en passant par les fils et les fibres.

Remerciements. nous remercions plus particulièrement le professeur P. Viallier, spécialiste de l'ennoblissement, pour les nombreuses et enrichissantes discussions que nous avons eues.

\section{Références}

[1] S. Ramakrishna, K. Fujihara, W.E. Teo, T.C. Lim, Z. $\mathrm{Ma}$, An introduction to electrospinning and nanofibers, World Scientific, 2005

[2] T.M. Sullivan, S. Middelman, Film thickness in blade coating of viscous and viscoelastic liquids, J.n.N.F.M. 21 (1986) 13-38

[3] T.M. Sullivan, S. Middelman, R. Keunings, Use of a finite element method to interpret rheological effects in blade coating, AIChE J. 33 (1987) 2047-2056

[4] E. Mitsoulis, T.V. Pham, Numerical simulation of rheological effects in blade coating flows, Proc. XIIth Int. Congr. Rheology (1996) 423-424, Ait-Kadi, Dealy, James, Williams (éd.) 
[5] F. Davard, D. Dupuis, Flow visualisation experiments in a blade coating process, J.n.N.F.M. 93 (2000) 17-28

[6] P. Doremus, J.M. Piau, Experimental study of viscoelastic effects in a cylinder-plane lubricated contact, J.n.N.F.M. 13 (1983) 79-91

[7] F. Davard, D. Dupuis, Blade coating of fabrics: rheology and fluid penetration, Coloration Technology 118 (2002) 69-74

[8] L.W.C. Miles, The production and properties of printing pastes in Textile Printing, in L.W.C. Miles (éd.), Society of Dyers and Colourists, 1994

[9] R. Lapasin, S. Pricl, Rheology of industrial polysaccharides: theory and applications, Blackie Academic and professional, Glasgow U.K., 1995

[10] J.L. Doublier, G. Cuvelier, Gums and hydrocolloids: functional aspects in Carbohydrates in food, A.C. Eliasson (éd.), Marcel Dekker Inc., New-York, 1996, pp. 283-318
[11] Z. Saffour, Étude des pâtes d'impression utilisées dans le domaine textile : rhéologie et morphologie des épaississants, thèse de doctorat, Mulhouse, 2007

[12] K.T. Nijenhuis, Thermoreversible networks, Advances in Polymer Science 130 (1997) 1-267

[13] S. Kesavan, R.K. Prud'homme, Rheology of guar and HPG cross-linked by borate, Macromolecules 25 (1992) 2026-2032

[14] J.O. Carnali, Gelation in physically associating biopolymer systems, Rheol. Acta 31 (1992) 399-412

[15] Z. Saffour, P. Viallier, D. Dupuis, Rheology of gel-like materials in textile printing, Rheol. Acta 45 (2006) 479-485

[16] S. Sejri, Influence du pré-mouillage sur la production et sur les caractéristiques mécaniques d'un fil encollé, Thèse de doctorat, Mulhouse 2008 